\title{
Cotunneling through a magnetic single-molecule transistor based on $\mathrm{N} @ \mathrm{C}_{60}$
}

\author{
Nicolas Roch, ${ }^{1}$ Romain Vincent, ${ }^{1}$ Florian Elste, ${ }^{2}$ Wolfgang Harneit, ${ }^{3}$ \\ Wolfgang Wernsdorfer, ${ }^{1}$ Carsten Timm, ${ }^{4}$ and Franck Balestro ${ }^{1}$ \\ ${ }^{1}$ Institut Néel, associé à l'UJF, CNRS, BP 166, 38042 Grenoble Cedex 9, France \\ ${ }^{2}$ Department of Physics, Columbia University, 538 West 120th Street, New York, NY 10027, USA \\ ${ }^{3}$ Institut für Experimentalphysik, Freie Universität Berlin, Arnimallee 14, 14195 Berlin, Germany \\ ${ }^{4}$ Institut für Theoretische Physik, Technische Universität Dresden, 01062 Dresden, Germany
}

(Dated: August 16, 2021)

\begin{abstract}
We present an experimental and theoretical study of a magnetic single-molecule transistor based on $\mathrm{N} @ \mathrm{C}_{60}$ connected to gold electrodes. Particular attention is paid to the regime of intermediate molecule-lead coupling, where cotunneling effects manifest themselves in the Coulomb-blockade regime. The experimental results for the differential conductance as a function of bias, gate voltage, and external magnetic field are in agreement with our analysis of the tunneling rates and provide evidence of magnetic signatures in single- $\mathrm{N} @ \mathrm{C}_{60}$ devices arising from an antiferromagnetic exchange interaction between the $\mathrm{C}_{60}$ spin and the nitrogen spin.
\end{abstract}

PACS numbers:

Single molecules carrying a magnetic moment, such as $\mathrm{Mn}_{12}, \mathrm{Fe}_{8}$ and $\mathrm{N}_{0} \mathrm{C}_{60}$, have been studied intensively for their outstanding quantum properties. Some of these molecules are characterized by a large spin and exhibit macroscopic quantum tunneling $[1,2]$ and quantuminterference effects [3]. The ability to initialize and manipulate the quantum state of a single magnetic molecule could open the road to new strategies for high-density information storage, quantum computing and molecular spintronics $[4,5]$. However, only few experiments have succeeded in fabricating single-molecule transistors (SMTs) [Fig. 1(a)] based on magnetic molecules [6-10]. Furthermore, only two experiments $[7,10]$ on single magnetic molecules in three-terminal geometry have succeeded to obtain the intermediate molecule-lead coupling regime. In this regime [11], cotunneling processes manifest themselves in the spectra of excited molecular states for a fixed charge state of the molecule, allowing an easier and more precise characterization of magnetic properties of single-molecule devices compared to sequential tunneling. For $\mathrm{Mn}_{12}$ clusters, steps in $d I / d V$ have been observed [7], but the dependence of the cotunneling steps on an external magnetic field was not investigated. Recently, a characteristic zero-field splitting and its evolution in an external magnetic field has been attributed to the signature of anisotropy in a $\mathrm{Fe}_{4}$ single-molecule magnet [10]. The aim of our work is then to study exchangecoupling-dependent cotunneling features in a single magnetic molecule.

Previous studies have shown that it is not straightforward to identify the magnetic properties of a SMT since molecular magnetism is usually destroyed during the device fabrication [7] due to strong interactions with the environment [12]. To conserve the magnetic properties, one option would be to incorporate a molecule whose magnetic moment is retained. In $\mathrm{N}_{0} \mathrm{C}_{60}$, the spin of the encapsulated nitrogen atom, which sits in the cen-

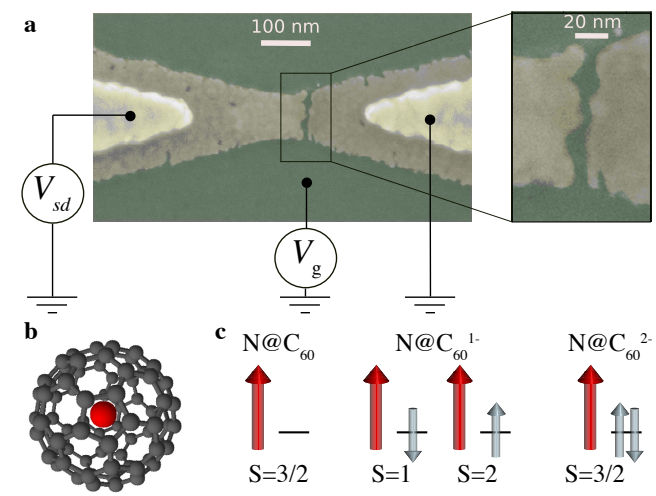

FIG. 1: (a) Colorized electron beam microscopy photography of an SMT consisting of a gold nanowire over an $\mathrm{Al} / \mathrm{Al}_{2} \mathrm{O}_{3}$ gate after electromigration. (b) Schematic picture of $\mathrm{N} @ \mathrm{C}_{60}$. (c) Spins states of neutral and charged $\mathrm{N} @ \mathrm{C}_{60}$.

ter of the fullerene molecule [13], is protected by the $\mathrm{C}_{60}$ cage [Fig. 1(b)]. An exchange interaction between the $\mathrm{C}_{60}$ spin and the nitrogen spin permits an indirect measurement of the magnetic properties of the nitrogen [9].

Although experimental studies investigating the stability of paramagnetism in $\mathrm{N} @ \mathrm{C}_{60}$ on a substrate have not been performed so far, it is known from X-ray and ultraviolet photoelectron spectroscopy that $\mathrm{C}_{60}$ changes its charge state when being adsorbed on noble metal surfaces $[14,15]$. This may lead to the formation of $\mathrm{N}_{6} \mathrm{C}_{60}^{1-}$ ions, when the number of electrons, $n_{\mathrm{C}_{60}}$, added to the $\mathrm{C}_{60}$ cage is 1 , or $\mathrm{N} @ \mathrm{C}_{60}^{2-}$ ions when $n_{\mathrm{C}_{60}}=2$. Since covalently functionalized $\mathrm{N} @ \mathrm{C}_{60}$ is stable [16-18], various ions of $\mathrm{N} @ \mathrm{C}_{60}$ are believed to be stable [19].

The total spin of neutral $\mathrm{N} @ \mathrm{C}_{60}$ is $S=3 / 2$ [Fig. 1.(c)]. However, the spin of the singly-charged ion $\mathrm{N} @ \mathrm{C}_{60}^{1-}$ can be $S=1$ or $S=2$, depending on whether the $\mathrm{C}_{60}$ spin $S_{\mathrm{C}_{60}}=1 / 2$ is antiparallel or parallel to the nitrogen spin [Fig. 1(c)]. N@ $\mathrm{C}_{60}^{2-}$ has a fixed ground state spin $S=3 / 2$ 
since the dianion cage is diamagnetic due to Jahn-Teller distortion.

Following theoretical predictions [20], transport measurements on SMTs with $\mathrm{N}_{0} \mathrm{C}_{60}$ in the weak-coupling regime showed signatures of an antiferromagnetic exchange interaction between the $\mathrm{C}_{60}$ spin and the nitrogen spin [9], in contradiction with the ferromagnetic exchange coupling obtained, for singly and triply ionized anions of $\mathrm{N} @ \mathrm{C}_{60}$, using multi-configurational self-consistent field methods [21]. Therefore, we first present results that confirm the interpretation of previous experiment [9] in the weak-coupling regime. As the field of molecular electronics in general and of molecular spintronics in particular has been plagued by a lack of reproducibility, it is essential that we have been able to reproduce the results for the prototypical single-molecule transistor based on $\mathrm{N} @ \mathrm{C}_{60}$ using independently fabricated devices. However, since we succeeded in reaching the intermediate molecule-lead coupling regime, our results go beyond the sequential-tunneling regime. In the second part, we present results in the cotunneling regime. In agreement with our theoretical analysis, our experimental results provide evidence for an antiferromagnetic exchange interaction between the $\mathrm{C}_{60}$ spin and the nitrogen spin.

The SMT was fabricated by using the electromigration technique [22]. The experiment was carried out in a dilution refrigerator at a base temperature of $35 \mathrm{mK}$. Our procedure and details of the measurement system are described in Ref. [23].

Figure 2(a) shows a color scale plot of the differential conductance $d I / d V$ as a function of bias voltage $V_{\text {sd }}$ and gate voltage $V_{\mathrm{g}}$ on the entire accessible gate range, performed during a first run of measurements. We emphasize that we observe only one charge degeneracy point (at gate voltage $V_{\mathrm{g}}^{\mathrm{D}}$ ), where the energy of the $n_{C_{60}}=1$ state aligns with energy of the $n_{C_{60}}=2$ state. Figure 2(b) shows results for $d I / d V$ in the vicinity of $V_{g}^{D}$. Analyzing the slopes of the stability diagram, we obtain $C_{g}$ : $C_{R}: C_{L}=1: 3.09: 2.96$ for the ratio of the capacitances, where $C_{g}, C_{R}$ and $C_{L}$ denote the capacitances of the gate, the source, and the drain with respect to the molecule. Thus the effective gate voltage is reduced by a factor of $C_{g} /\left(C_{g}+C_{R}+C_{L}\right)=0.142$. From this we infer that the energy spacing between the relevant charge degeneracy points is at least larger than $230 \mathrm{meV}$ and conjecture that the measurement was performed for a single $\mathrm{N} @ \mathrm{C}_{60}$ in the junction [24].

When the energy level spacing between the molecular states is large compared to the thermal energy, the hybridization energies $\Gamma_{R}$ and $\Gamma_{L}$ can be estimated by an analysis of the current amplitudes at different gate voltages [25]. We find $\Gamma_{L} \simeq 1.65 \mu \mathrm{eV}$ and $\Gamma_{R} \simeq 16.5 \mu \mathrm{eV}$ $\left(\Gamma_{R} / \Gamma_{L} \simeq 10\right)$. The assumption of asymmetric moleculelead couplings explains the different peak heights in $d I / d V$ at negative and positive bias [Fig. 2(b)] and is taken into account in the theoretical treatment discussed

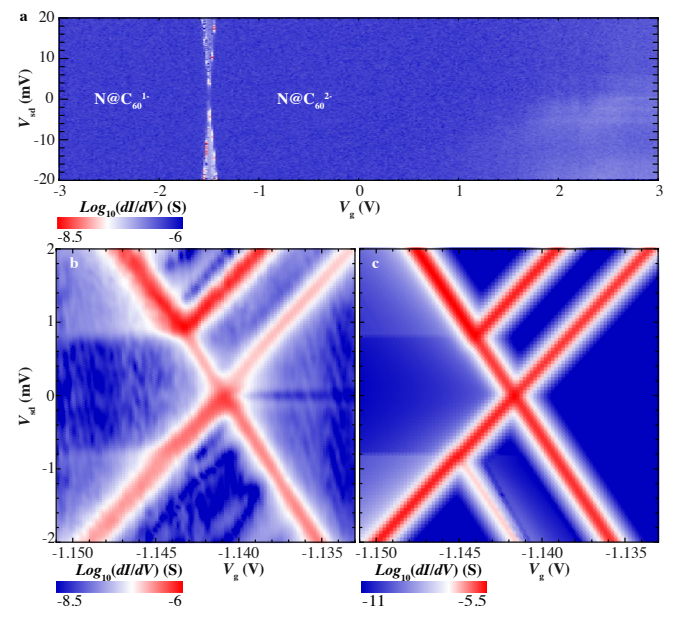

FIG. 2: Color scale plot of the differential conductance $d I / d V$ as a function of bias voltage $V_{\mathrm{sd}}$ and gate voltage $V_{\mathrm{g}}$ (a) on the entire accessible $V_{\mathrm{g}}$ range and (b) in the vicinity of $V_{\mathrm{g}}^{\mathrm{D}}$. (c) Numerical results obtained from Eqs. (4) and (5) for $\varepsilon_{d}=$ $-3.1625 \mathrm{eV}, U=3.0 \mathrm{eV}, J=-0.4 \mathrm{meV}, C_{g}: C_{R}: C_{L}=1$ : $3.09: 2.96$, and $\Gamma_{R} / \Gamma_{L} \simeq 10$.

below [Fig. 2(c)].

We compare the bias- and gate-voltage dependences of the measured differential conductance to numerical results obtained from a rate-equation approach. The system is described by a Hamiltonian of the form $H=$ $H_{\mathrm{N} @ \mathrm{C}_{60}}+H_{\text {leads }}+H_{t}$, where

$$
\begin{aligned}
H_{\mathrm{N} \mathrm{C}_{60}}= & \varepsilon_{d} n_{\mathrm{C}_{60}}+\frac{U}{2} n_{\mathrm{C}_{60}}\left(n_{\mathrm{C}_{60}}-1\right) \\
& -J \mathbf{S}_{\mathrm{C}_{60}} \cdot \mathbf{S}_{\mathrm{N}}-g \mu_{B} B\left(S_{\mathrm{C}_{60}}^{z}+S_{\mathrm{N}}^{z}\right)
\end{aligned}
$$

describes the molecular degrees of freedom,

$$
H_{\text {leads }}=\sum_{\alpha=L, R} \sum_{\mathbf{k} \sigma} \epsilon_{\alpha \mathbf{k}} a_{\alpha \mathbf{k} \sigma}^{\dagger} a_{\alpha \mathbf{k} \sigma}
$$

describes the conduction electrons in the leads, and

$$
H_{t}=\sum_{\alpha=L, R} \sum_{\mathbf{k} \sigma}\left(t_{\alpha} a_{\alpha \mathbf{k} \sigma}^{\dagger} d_{\sigma}+t_{\alpha}^{*} d_{\sigma}^{\dagger} a_{\alpha \mathbf{k} \sigma}\right)
$$

describes the tunneling of electrons between the $\mathrm{C}_{60}$ cage and the leads. Here, $d_{\sigma}^{\dagger}$ creates an electron with spin $\sigma$ and energy $\varepsilon_{d}$ in the LUMO of the $\mathrm{C}_{60}$ molecule, while $a_{\alpha \mathbf{k} \sigma}^{\dagger}$ creates an electron in lead $\alpha$ with spin $\sigma$, momentum $\mathbf{k}$, and energy $\epsilon_{\alpha \mathbf{k}}$. Tuning the gate voltage $V_{\mathrm{g}}$ allows one to shift the orbital energy $\varepsilon_{d}$. The number and spin operator of the $\mathrm{C}_{60}$ electrons are $n_{\mathrm{C}_{60}}=\sum_{\sigma} d_{\sigma}^{\dagger} d_{\sigma}$ and $\mathbf{S}_{\mathrm{C}_{60}}=\sum_{\sigma \sigma^{\prime}} d_{\sigma}^{\dagger}\left(\boldsymbol{\sigma}_{\sigma \sigma^{\prime}} / 2\right) d_{\sigma^{\prime}}$, respectively, with $\boldsymbol{\sigma}$ the vector of the Pauli matrices. The exchange interaction with the nitrogen spin $\mathbf{S}_{\mathrm{N}}$ is denoted by $J$ and the external magnetic field applied along the $z$ axis by $B$, where $g$ is the $g$-factor and $\mu_{B}$ is the Bohr magneton.

The leading contribution to the tunneling rates (corresponding to sequential tunneling) is of second order in 
$H_{t}$ and can be obtained from Fermi's Golden Rule,

$$
\begin{aligned}
R_{n \rightarrow n^{\prime}, \alpha}^{(\mathrm{seq})}= & \Gamma_{\alpha} \sum_{\sigma}\left(f\left(\epsilon_{n^{\prime}}-\epsilon_{n}-\mu_{\alpha}\right)\left|C_{n n^{\prime}}^{\sigma}\right|^{2}\right. \\
& \left.+\left[1-f\left(\epsilon_{n}-\epsilon_{n^{\prime}}-\mu_{\alpha}\right)\right]\left|C_{n^{\prime} n}^{\sigma}\right|^{2}\right) .
\end{aligned}
$$

Here $n$ and $n^{\prime}$ denote the initial and final many-particle state of $\mathrm{N}_{0} \mathrm{C}_{60}$, and $f$ is the Fermi function. The matrix element with $C_{n n^{\prime}}^{\sigma} \equiv\left\langle n\left|d_{\sigma}\right| n^{\prime}\right\rangle$ denotes the net overlap of the initial and final states of the molecule. The typical sequential-tunneling rate is given by $\Gamma_{\alpha}=2 \pi t_{\alpha} 2 \nu_{\alpha}$, where $\nu_{\alpha}$ denotes the lead density of states $(\hbar=1)$. To mimick the finite life-time broadening of the sequentialtunneling peaks, we used an effective temperature of $250 \mathrm{mK}$ in Eq. (4).

The next-to-leading-order contribution to the tunneling rates (including cotunneling) is of fourth order in $H_{t}$ and can be computed using a $T$-matrix formalism $[26,27]$. We find

$$
\begin{aligned}
R_{n \rightarrow n^{\prime}, \alpha \rightarrow \alpha^{\prime}}^{(\cot )} & =\frac{\Gamma_{\alpha} \Gamma_{\alpha^{\prime}}}{2 \pi} \sum_{\sigma \sigma^{\prime}} \int d \epsilon\left\{\left|\sum_{n^{\prime \prime}} \frac{C_{n^{\prime \prime} n^{\prime}}^{\sigma *} C_{n^{\prime \prime} n}^{\sigma^{\prime}}}{\epsilon_{n^{\prime}}-\epsilon_{n^{\prime \prime}}-\epsilon+i \eta}\right|^{2}\right. \\
& \left.+\left|\sum_{n^{\prime \prime}} \frac{C_{n^{\prime} n^{\prime \prime}}^{\sigma} C_{n n^{\prime \prime}}^{\sigma^{\prime} *}}{\epsilon_{n}-\epsilon_{n^{\prime \prime}}+\epsilon+i \eta}\right|^{2}\right\} \\
& \times f\left(\epsilon-\mu_{\alpha}\right)\left[1-f\left(\epsilon_{n}-\epsilon_{n^{\prime}}+\epsilon-\mu_{\alpha^{\prime}}\right)\right]
\end{aligned}
$$

The divergences arising from the energy denominators are regularized by replacing $\eta$ by a finite life-time broadening $\Gamma=\Gamma_{L}+\Gamma_{R}$. In the Coulomb-blockade regime far from the sequential-tunneling threshold, the Fermi functions suppress the integrand in Eq. (5) outside of a narrow energy interval. Replacing $\epsilon$ in the energy denominators by its mean on these intervals, we can perform the integral. Close to the sequential-tunneling threshold, where this approximation becomes invalid, the cotunneling contribution is overwhelmed by the sequential tunneling in any case.

The good qualitative agreement between Figs. 2(b) and 2(c) suggests that the Hamiltonian [Eqs. (1)-(3)] contains the crucial ingredients to explain both sequential and cotunneling features revealed in the experiment. We find that a calculation under the assumption of $J \simeq-0.4 \mathrm{meV}$ gives the best agreement with the experiment.

In order to verify that the charge degeneracy point involves the two states $n_{\mathrm{C}_{60}}=1$ and $n_{\mathrm{C}_{60}}=2$, we investigated the dependence of $d I / d V$ on an external magnetic field $B$. These measurements were performed for the same sample but during a different run. Results of $d I / d V$ for a fixed gate voltage greater than $V_{\mathrm{g}}^{\mathrm{D}}$ are shown in Fig. 3. The conductance peaks at positive and negative bias voltage first move apart until the magnetic field assumes a critical value. Then they change slope approaching each other again. Here the best fit to experimental data is obtained for a calculation that assumes $J \simeq-0.3 \mathrm{meV}$. The other parameters are also changed

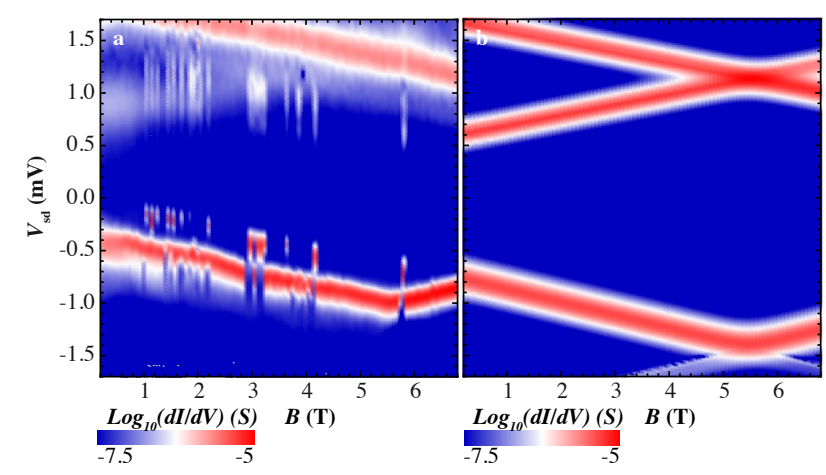

FIG. 3: Color scale plot of the differential conductance $d I / d V$ as a function of bias voltage and magnetic field $B$. (a) shows experimental results while (b) shows numerical results in the regime where the ground state is $n_{\mathrm{C}_{60}}=2$, with $\varepsilon_{d}=-3.1722 \mathrm{eV}, U=3.0 \mathrm{eV}, J=-0.3 \mathrm{meV}, C_{g}: C_{R}$ : $C_{L}=1: 3.29: 3.43$, and $\Gamma_{R} / \Gamma_{L} \simeq 6.7$.

somewhat, see the caption of Fig. 3. The microscopic mechanism behind the change in the exchange interaction is not yet clear. The characteristic pattern revealed in Fig. 3 allows us to deduce the charge and spin of the relevant molecular states to the left and to the right of the degeneracy point, in agreement with the results reported by Grose et al. [9].

We now turn to the cotunneling regime where transport is dominated by the coherent tunneling of electrons through the source-molecule-drain structure [28, 29]. This regime is characterized by a fixed molecular charge state and by the occurrence of steps in $d I / d V$. The positions of these steps are independent of the molecular charging energies and the gate voltage but do depend on the magnetic excitation energies. As a consequence, measurements in this regime can be more easily interpreted than in the sequential-tunneling regime, where two different charge states are involved.

For $\mathrm{N} @ \mathrm{C}_{60}^{1-}$, the ground state is a degenerate spin $S=1$ multiplet while the excited state, having an energy $J$ compared to the ground state, is a spin $S=2$ multiplet at zero magnetic field. Turning on a magnetic field leads to a Zeeman splitting of the spin multiplets as indicated in Fig. 4(a). Selection rules for the cotunneling processes require $\left|\Delta S^{z}\right|=0$ or 1 . This condition is used to determine all possible transitions that we expect to observe as steps in $d I / d V$ (Fig. 4(b)). Our measurement presented in Fig. 4(c) is in qualitative agreement with our theoretical analysis presented in Fig. 4(d), obtained with the same exchange coupling $J=-0.3 \mathrm{meV}$, confirming in an another tunneling process, the antiferromagnetic exchange coupling between the $\mathrm{C}_{60}$ spin and the nitrogen spin.

The discussion for the case $V_{g}>V_{g}^{D}$, where the ground state is a doubly-charged $\mathrm{N} @ \mathrm{C}_{60}^{2-}$ anion, proceeds in an analogous way and is included in the supplementary in- 

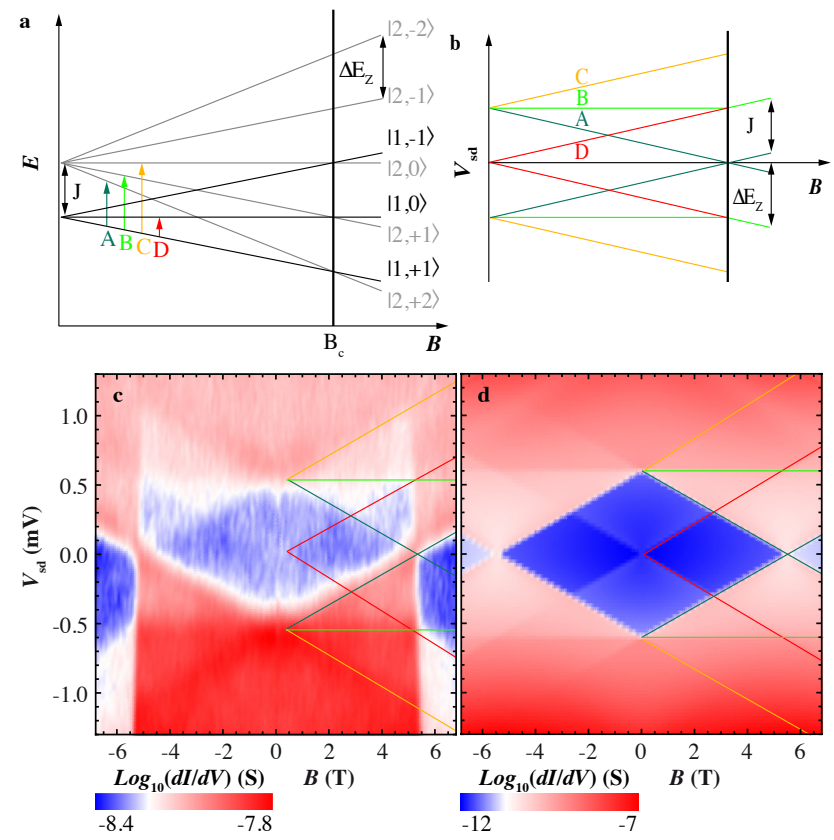

FIG. 4: (a) Zeeman diagram of the multiplets $S=1$ and $S=2$ for $\mathrm{N} @ \mathrm{C}_{60}^{1-}\left(n_{\mathrm{C}_{60}}=1\right)$. (b) Schematic showing the expected cotunneling steps. (c) Experimental and (d) numerical results, with $J=-0.3 \mathrm{meV}$, of $d I / d V$ for a fixed gate voltage $V_{\mathrm{g}}<V_{\mathrm{g}}^{\mathrm{D}}$. Colored lines indicate the positions of the magnetic excitations illustrated in (a) and (b).

formation. The most striking difference is the presence of a cotunneling step at very low bias, for zero magnetic field [Fig. 2(b)]. This feature might be due to magnetic anisotropy of the molecular spin [10], caused by the spinorbit coupling of the nitrogen, which we expect to be enhanced by the hybridization with the gold leads.

In summary, we have presented an experimental and theoretical analysis of an SMT based on $\mathrm{N} @ \mathrm{C}_{60}$. Our results in the sequential-tunneling regime are in agreement with the findings previously reported [9], and are a step towards achieving the high degree of reproducibility necessary for progress in the field of molecular spintronics. Furthermore, we have investigated the cotunneling regime, where transport is dominated by the coherent transfer of electrons through the molecular junction. Since measurements in this regime can be more easily interpreted than in the sequential-tunneling regime, the characteristic two-dimensional plots of $d I / d V$ as a function of bias voltage and magnetic field serve a characteristic fingerprint of the magnetic state. In particular, cotunneling data obtained as a function of magnetic field corroborate the antiferromagnetic exchange interaction between $\mathrm{C}_{60}$ spin and the nitrogen spin.

We thank E. Eyraud, D. Lepoittevin for technical support, T. Crozes, T. Fournier for help with the lithographic methods, S. Florens, V. Bouchiat, C. Winkelmann, L. Udvardi for fruitful discussions, and C. Thirion, E. Bonet, R. Piquerel for help with the software development. Samples were fabricated in the NANOFAB facility of the Néel Institute. This work is financially supported by ANR-PNANO project MolNanoSpin n ${ }^{\circ}$ ANR-08-NANO-002, ERC Advanced Grant MolNanoSpin $\mathrm{n}^{\circ} 226558$, STEP MolSpinQIP, Cible 2009, and the Deutsche Forschungsgemeinschaft.

[1] J. R. Friedman, M. P. Sarachik, J. Tejada, and R. Ziolo, Phys. Rev. Lett. 76, 3830 (1996).

[2] L. Thomas et al., Nature (London) 383, 145 (1996).

[3] W. Wernsdorfer and R. Sessoli, Science 284, 133 (1999).

[4] W. Harneit, Phys. Rev. A 65, 032322 (2002).

[5] L. Bogani and W. Wernsdorfer, Nature Materials 7, 179 (2008).

[6] H. B. Heersche et al., Phys. Rev. Lett. 96, 206801 (2006).

[7] M. H. Jo et al., Nanoletters 6, 2014 (2006).

[8] J. J. Henderson, C. M. Ramsey, E. D. Barco, A. Mishra, and G. Christou, J. Appl. Phys. 101, 09E102 (2007).

[9] J. E. Grose et al., Nature Materials 7, 884 (2008).

[10] A. S. Zyazin et al., Nanoletters 10, 3307 (2010).

[11] K. Moth-Poulsen and T. Bjørnholm, Nature Nanotechnology 4, 551 (2009).

[12] M. Mannini et al. Chemistry-A European Journal, 14, 7530 (2008).

[13] H. Mauser et al., Angew. Chem. Int'l. Ed. 36, 2835 (1997).

[14] S. Modesti, S. Cerasari, and P. Rudolf, Phys. Rev. Lett. 71, 2469 (1993).

[15] N. Swami, H. He, and B. E. Koel, Phys. Rev. B 59, 8283 (1999).

[16] E. Dietel et al., J. Am. Chem. Soc. 121, 2432 (1999).

[17] B. Goedde et al., Chem. Phys. Lett. 334, 12 (2001).

[18] L. Franco et al., Chem. Phys. Lett. 422, 100 (2006).

[19] P. Jakes et al., in Electronic Properties of NovelMaterials-Molecular Nanostructures, edited by H. Kuzmany et al., AIP Conf. Proc. 544, 174 (AIP, Melville, NY, 2000).

[20] F. Elste and C. Timm, Phys. Rev. B 71, 155403 (2005).

[21] L. Udvardi, in Electronic Properties of Novel-MaterialsMolecular Nanostructures, edited by H. Kuzmany et al., AIP Conf. Proc. 544, 187 (AIP, Melville, NY, 2000).

[22] H. Park, A. K. L. Lim, A. P. Alivisatos, J. Park, and P. L. McEuen, Appl. Phys. Lett. 75, 301 (1999).

[23] N. Roch, S. Florens, V. Bouchiat, W. Wernsdorfer, and F. Balestro, J. Low Temp. Phys. 153, 350 (2008).

[24] D. R. Ward, G. D. Scott, Z. K. Keane, N. J. Halas, and D. Natelson, J. Phys. : Cond. Matter 20, 374118 (2008).

[25] E. Bonet, M. M. Deshmukh, and D. C. Ralph, Phys. Rev. B 65, 45317 (2002).

[26] H. Bruus and K. Flensberg, in Many-body Quantum Theory in Condensed Matter Physics, (Oxford University Press, Oxford, 2004).

[27] F. Elste and C. Timm, Phys. Rev. B 75, 195341 (2007).

[28] D. V. Averin and Y. V. Nazarov, in Single Charge Tunneling: Coulomb Blockade phenomena in Nanostructures, edited by H. Grabert and M. H. Devoret (Plenum Press and NATO Scientific Affairs Division, New York, 1992) p 217.

[29] S. De Franceschi et al., Phys. Rev. Lett. 86, 878 (2001). 


\title{
Supplementary information : Cotunneling through a magnetic single-molecule transistor based on $\mathrm{N} @ \mathrm{C}_{60}$
}

\author{
Nicolas Roch, ${ }^{1}$ Romain Vincent, ${ }^{1}$ Florian Elste, ${ }^{2}$ Wolfgang Harneit, ${ }^{3}$ \\ Wolfgang Wernsdorfer, ${ }^{1}$ Carsten Timm, ${ }^{4}$ and Franck Balestro ${ }^{1}$ \\ ${ }^{1}$ Institut Néel, associé à l'UJF, CNRS, \\ BP 166, 38042 Grenoble Cedex 9, France \\ ${ }^{2}$ Department of Physics, Columbia University, \\ 538 West 120th Street, New York, NY 10027, USA \\ ${ }^{3}$ Institut für Experimentalphysik, Freie Universität Berlin, \\ Animallee 14, 14195 Berlin, Germany \\ ${ }_{4}^{4}$ Institut für Theoretische Physik, Technische Universität Dresden, 01062 Dresden, Germany \\ PACS numbers:
}




\section{SAMPLES PRESENTATION AND COMPARISON OF THE RESULTS}

Single-molecule transistors (SMT's) based on $\mathrm{N} @ \mathrm{C}_{60}$ contacted by gold leads were prepared by blow drying a dilute toluene solution of the $\mathrm{N} @ \mathrm{C}_{60}$ molecule onto a gold nano-wire realized on an $\mathrm{Al} / \mathrm{Al}_{2} \mathrm{O}_{3}$ back gate. Before blow drying the solution, the nano-wires were cleaned with acetone, ethanol, isopropanol and oxygen plasma. We then performed our electromigration procedure at $4 \mathrm{~K}$. We tested 274 junctions and present in this section 3 samples having an addition energy greater than 200 meV (Fig.S.1)
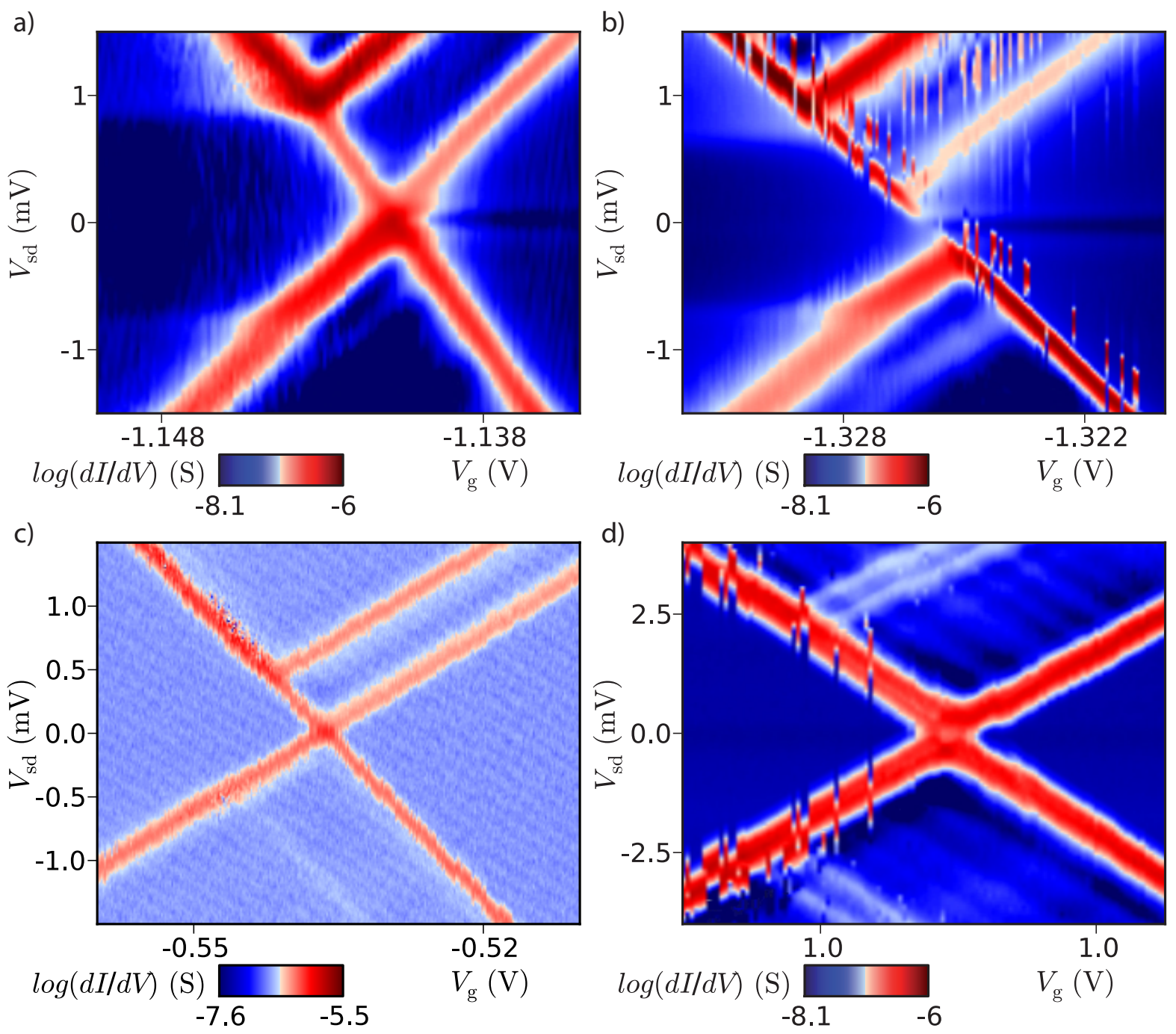

Fig.S. 1: Color scale plot of the differential conductance $d I / d V$ as a function of bias voltage $V_{\text {sd }}$ and gate voltage $V_{\mathrm{g}}$ : a) first run of measurements. b) second run of measurements. c) and d) other $\mathrm{N} @ \mathrm{C}_{60}$ SMT's in the weak coupling regime. 
In Fig.S.1(a) and 1(b) we present first the stability diagram of the sample presented in the Letter, obtained at zero magnetic field during the first and the second run of measurements, respectively. The sample has been kept at cryogenic temperature $(T \leq 4 \mathrm{~K})$ during the two runs. However, changes in our electronic setup (different I-V converters, inversion of the ground connection) have probably generated a small electrical discharge inducing modifications of some measurement features, as exhibited by the modification of the capacitances and tunneling rates extracted from a fine analysis of the stability diagram. For the first run, we obtain $C_{g}: C_{R}: C_{L}=1: 3.09: 2.96$, and $\Gamma_{R} / \Gamma_{L} \simeq 10$, while for the second run we get $C_{g}: C_{R}: C_{L}=1: 3.29: 3.43$, and $\Gamma_{R} / \Gamma_{L} \simeq 6.7$. The SMT experienced then some unexpected changes inducing a different electrostatic environment leading to a modification of the anti-ferromagnetic exchange interaction $J$. The evolution of $J$ will be addressed in the next section.

The striking difference between the two runs is observed at the charge degeneracy point. In Fig.S.1(b), corresponding to the second run, we measure an opening of this latter. As it is not present in the first run as seen in Fig.S.1(a), we can rule out any correlation between this observation and the cotunneling step at low bias voltage observed for $n_{\mathrm{C}_{60}}=2\left(V_{\mathrm{g}}>V_{\mathrm{g}}^{\mathrm{D}}\right)$. No further measurements were performed in order to explain this feature, but a similar behavior was observed in another SMT based on a single $\mathrm{N} @ \mathrm{C}_{60}$, as presented in Fig.S.1(d).

\section{MAGNETIC FIELD SPECTROSCOPY IN THE COTUNNELING REGIME FOR} $n_{\mathrm{C}_{60}}=1$

In this section, we compare the magnetic field spectroscopy performed in the cotunneling regime, where $n_{\mathrm{C}_{60}}=1\left(V_{\mathrm{g}}<V_{\mathrm{g}}^{\mathrm{D}}\right)$, for the two runs of measurements. As depicted in Figure 4 of the Letter, when the magnetic field increases, the different multiplet states are Zeeman

split. For a magnetic field $B<B_{\mathrm{c}}$, the ground sate is $|1,+1\rangle$ and taking into account the selection rules, we observe four different transitions, until the magnetic field reaches the critical magnetic field $B_{\mathrm{c}}$ for which the ground state becomes $|2,+2\rangle$. Only two transitions are then allowed, obeying $\left|\Delta S^{z}\right|=0$ or 1, as observed in Fig.S.2(a). Unfortunately, if the critical field $B_{\mathrm{c}}$ was experimentally accessible for the second run corresponding to $J=$ -0.3 meV (Fig.S.2(a)), no spin transition could be observed for the first run (Fig.S.2(b)) as the value of $J=-0.4 \mathrm{meV}$ was too high. Indeed, as $J$ depends exponentially on the distance 

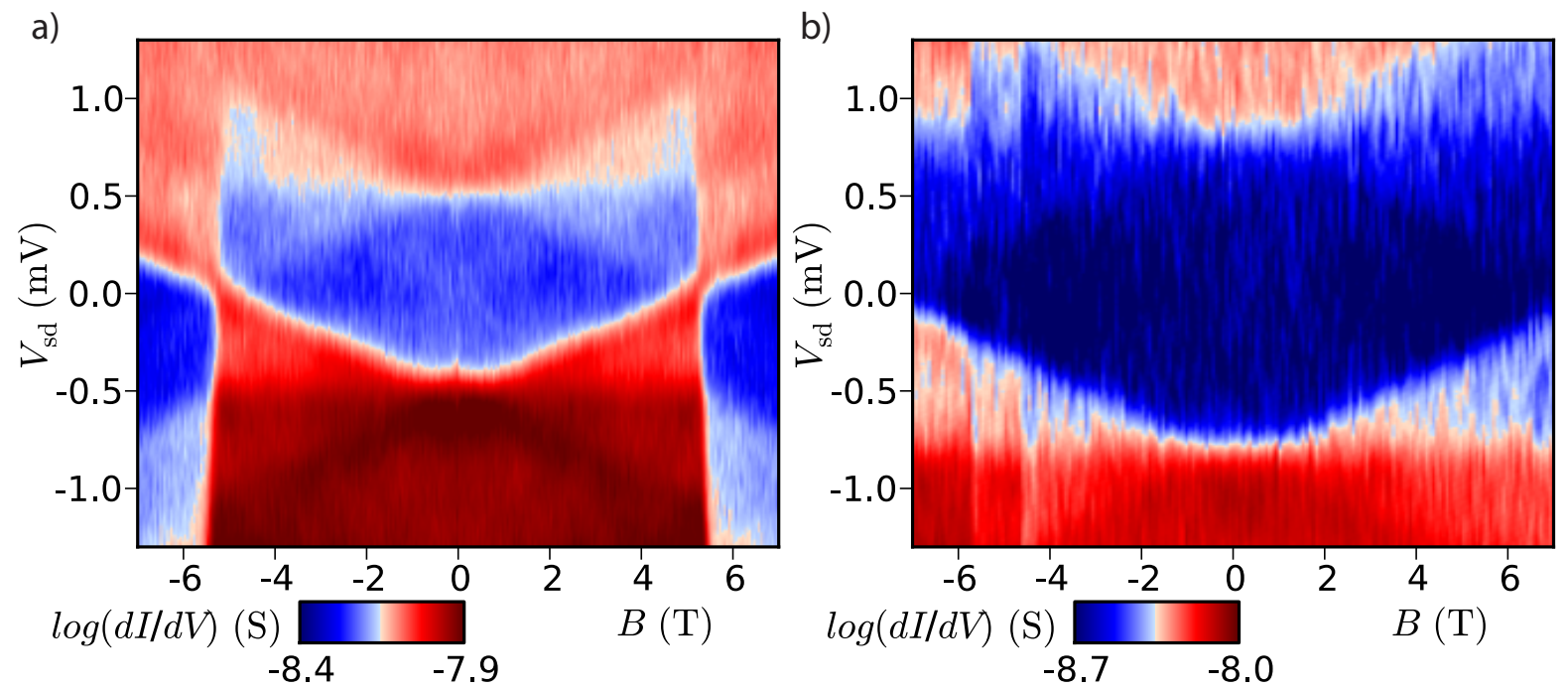

Fig.S. 2: Experimental differential conductance as a function of magnetic field for a fixed gate voltage $V_{\mathrm{g}}<V_{\mathrm{g}}^{\mathrm{D}}\left(n_{\mathrm{C}_{60}}=1\right)$ for (a) $J=-0.3 \mathrm{meV}$ (second run) and (b) $J=-0.4 \mathrm{meV}$ (first run).

between the nitrogen and the $\mathrm{C}_{60}$ cage, a small deformation can modify $J$ significantly. More precisely, $J$ is given by an exchange integral, which depends strongly on the electronic wave function of the $\mathrm{C}_{60}$ LUMO. This wave function is not only changed by geometric deformation but also by electrostatics. For example, image charges in the metal electrodes draw it outwards towards the electrodes and away from the nitrogen. A modification of the environment can thus also lead to a variation of $J$.

\section{MAGNETIC FIELD SPECTROSCOPY IN THE COTUNNELING REGIME FOR} $n_{\mathrm{C}_{60}}=2$

In the Letter, we raised the question of the unexpected cotunneling steps at low voltage bias, obtained at zero magnetic field in the $n_{\mathrm{C}_{60}}=2$ region where the total spin of $\mathrm{N} @ \mathrm{C}_{60}^{2-}$ is $S=3 / 2$. In Eq. (1) of the Letter, we assume no anisotropy. In this case, performing a magnetic field spectroscopy, we should observe one cotunneling step increasing linearly with magnetic field (Fig.S.3(b)) corresponding to the transition from the $|3 / 2,+3 / 2\rangle$ state to the $|3 / 2,+1 / 2\rangle$ state as shown in Fig.S.3(a). In Fig.S.3(c), we present the $d I / d V$ measurement as a function of magnetic field. We clearly observe that the cotunneling steps evolve linearly 
a)
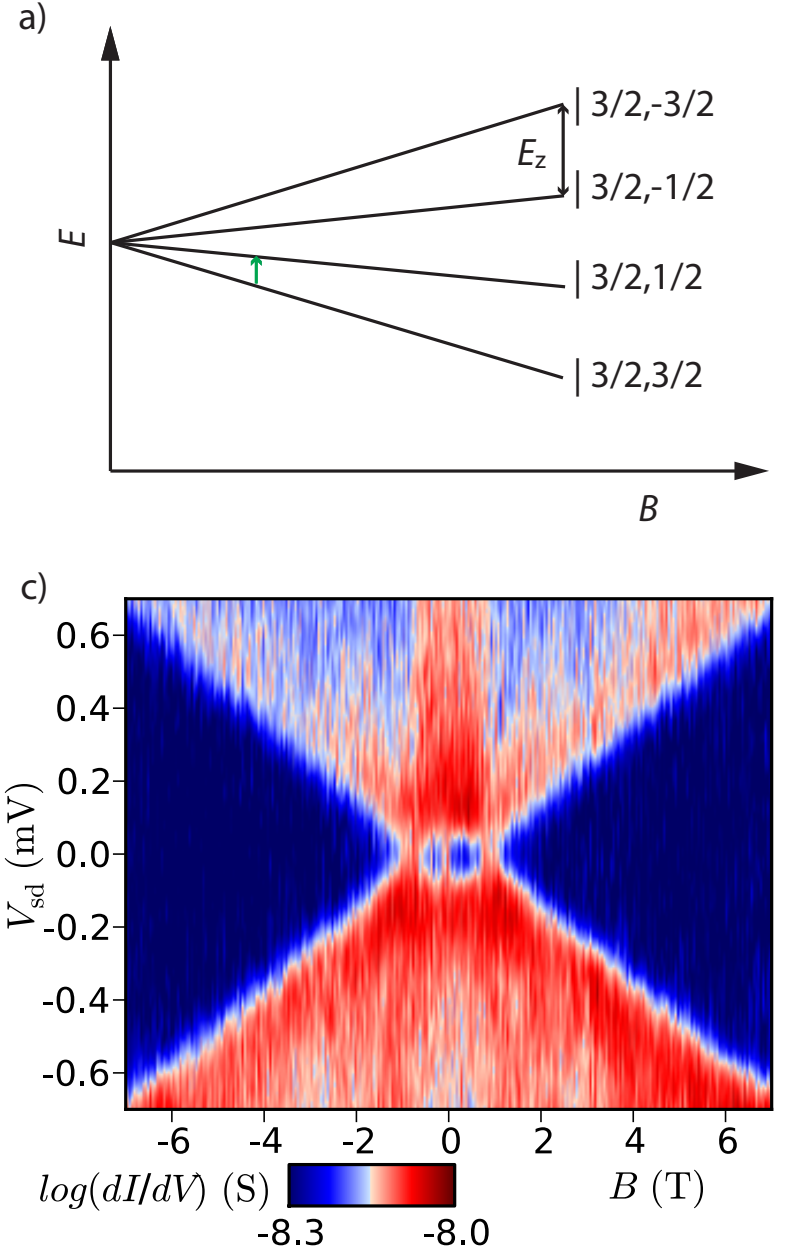

b)
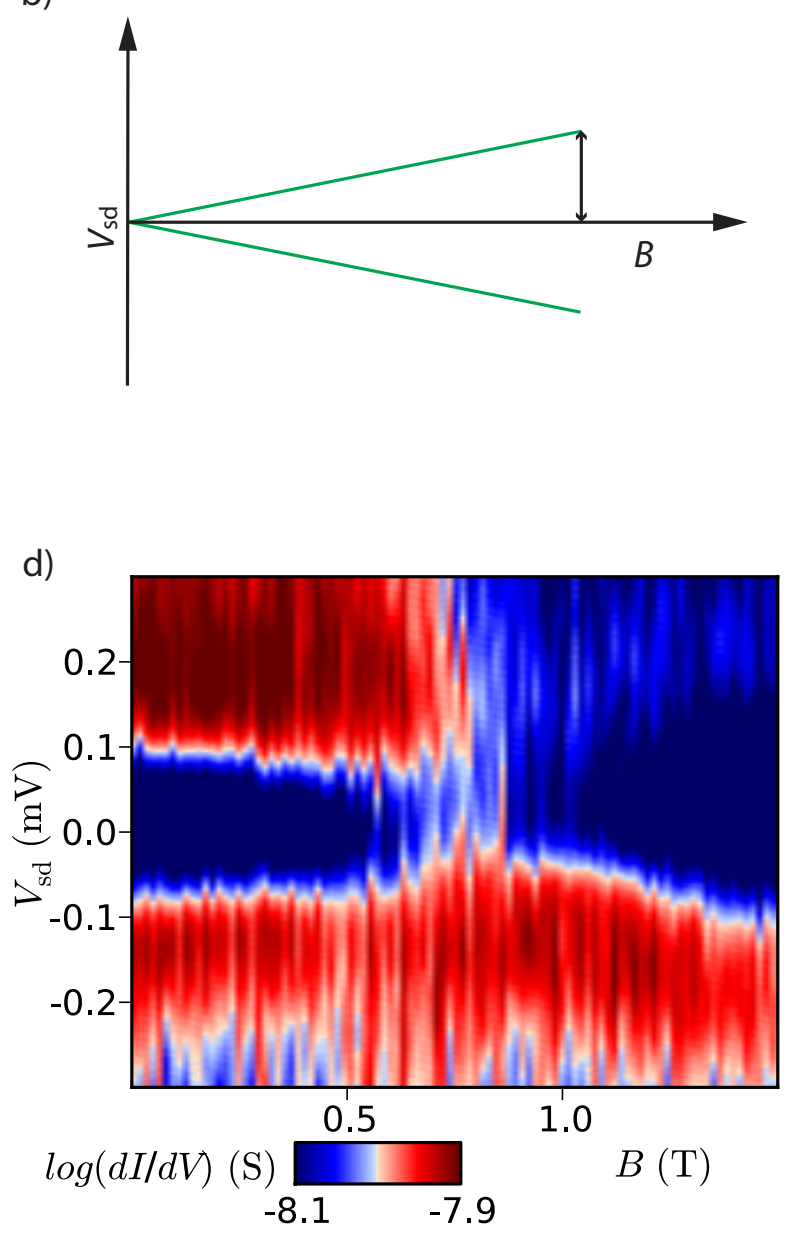

Fig.S. 3: (a) Zeeman diagram of the multiplet $S=3 / 2$ for $\mathrm{N}_{0} \mathrm{C}_{60}^{2-} \quad\left(n_{\mathrm{C}_{60}}=2\right)$. (b) Schematic showing the expected cotunneling steps deduced from the Zeeman diagram. (c) and (d) $d I / d V$ measurements as a function of magnetic field for a fixed gate voltage.

in the high magnetic field limit.

However, when we focus on the low magnetic field spectroscopy measurements (Fig.S.3(d)), we observe a non-linear decrease of the cotunneling step as a function of magnetic field. This cotunneling step and the non linear evolution could be interpreted as a signature of anisotropy [1-5] in $\mathrm{N} @ \mathrm{C}_{60}^{2-}$. This interpretation relies on the assumption of significantly enhanced spin-orbit coupling on the nitrogen atom, which might be caused by the hybridization with the gold leads, as the anisotropy of $\mathrm{N} @ \mathrm{C}_{60}$ should not exceed tens of $\mathrm{mT}[6-8]$.

The effect of anisotropy can be included in our model adding $E_{\text {ani }}=-K\left(S^{z}\right)^{2}$ in our Hamiltonian (Eq. 1 of the Letter), where $E_{\text {ani }}$ is the anisotropic energy, $K$ is the uniaxial 
anisotropy constant, and $S_{z}$ is the z component of the total spin. However, by including this term into the Hamiltonian, we should also observe extra cotunneling steps in the $n_{\mathrm{C}_{60}}=1$ region. We did not measured this signature, either because of a lack of sensitivity of our electronic, or because the finite anisotropy might be charge dependent, but up to now, we do not have a clear explanation of the possible charging-state dependence of $K$.

[1] M. H. Jo, J. E. Grose, K. Baheti, M. M. Deshmukh, J. J. Sokol, E. M. Rumberger, D. N. Hendrickson, J. R. Long, H. Park, and D. C. Ralph, Nanoletters 6, 2014 (2006).

[2] H. B. Heersche, Z. de Groot, J. A. Folk, H. S. J. van der Zant, C. Romeike, M. R. Wegewijs, L. Zobbi, D. Barreca, E. Tondello, and A. Cornia, Phys. Rev. Lett. 96, 206801 (2006).

[3] A. S. Zyazin, J. W. G. van den Berg, E. A. Osorio, H. S. J. van der Zant, N. P. Konstantinidis, M. Leijnse, M. R. Wegewijs, F. May, W. Hofstetter, C. Danieli, and A. Cornia, Nanoletters 10, 3307 (2010).

[4] C. Romeike, M. R. Wegewijs, and H. Schoeller, Phys. Rev. Lett. 96, 196805 (2003).

[5] C. Timm, Phys. Rev. B 76, 014421 (2003).

[6] K. Lips, M. Waiblinger, B. Pietzak, and A. Weidinger, Physica Status Solidi (a), 177, 81 (2000).

[7] L. Franco, S. Ceola, C. Corvaja, S. Bolzonella, W. Harneit, and M. Maggini, Chem. Phys. Lett. 422, 100, (2006)

[8] B. Naydenov, C. Spudat, W. Harneit, H. I. Süss, J. Hulliger, J. Nuss, and M. Jansen, Chem. Phys. Lett. 424, 327, (2006). 